\title{
Surface-to-Surface Registration Using Level Sets
}

\author{
Mads Fogtmann Hansen ${ }^{1}$, Søren Erbou ${ }^{1}$, Martin Vester-Christensen ${ }^{1}$, \\ Rasmus Larsen ${ }^{1}$, Bjarne Ersbøll ${ }^{1}$, and Lars Bager Christensen ${ }^{2}$ \\ 1 Technical University of Denmark \\ ${ }^{2}$ Danish Meat Research Institute
}

\begin{abstract}
This paper presents a general approach for surface-to-surface registration (S2SR) with the Euclidean metric using signed distance maps. In addition, the method is symmetric such that the registration of a shape A to a shape B is identical to the registration of the shape $\mathrm{B}$ to the shape A.

The S2SR problem can be approximated by the image registration (IR) problem of the signed distance maps (SDMs) of the surfaces confined to some narrow band. By shrinking the narrow bands around the zero level sets the solution to the IR problem converges towards the S2SR problem. It is our hypothesis that this approach is more robust and less prone to fall into local minima than ordinary surface-to-surface registration. The IR problem is solved using the inverse compositional algorithm.

In this paper, a set of 40 pelvic bones of Duroc pigs are registered to each other w.r.t. the Euclidean transformation with both the S2SR approach and iterative closest point approach, and the results are compared.
\end{abstract}

\section{Introduction}

This paper addresses the problem of shape registration or alignment which plays an essential role in shape analysis. Many registration procedures such as generalized Procrustes analysis [9]7 rely on a prior manual annotation of landmarks. The main drawback with these approaches is the reliance on manual annotation which becomes cumbersome and infeasible for larger 2Ddatasets and for 3D data.

Methods for explicitly deriving landmarks form training curves/surfaces based on information theoretic theory has been published [6]. Unfortunately these often suffer form exceeding use of computation time.

The iterative closest point (ICP) algorithm by Besl et al. 2] solves the problem of landmark dependence by iteratively updating the point correspondence after the closest point criterium. Since the introduction in 1992 many extensions and improvements of original ICP have been proposed in literature 8[11]10. Most of these methods still require a good initial estimate in order not to converge to a local minimum. Furthermore, common for these methods are that they do not utilize the knowledge of the connectedness of the point cloud, which is available in many cases.

B.K. Ersbøll and K.S. Pedersen (Eds.): SCIA 2007, LNCS 4522, pp. $780788,2007$.

(C) Springer-Verlag Berlin Heidelberg 2007 
The approach described in this paper is in many ways related to the approach presented by Darkner et al. [5], which aligns two point clouds by minimizing the sum of squared difference between the distance functions of the point clouds in some rectangular box domain. The problem with the scheme by Darkner et al. is that it is likely produce a suboptimal result when applied to concave shapes. That is, the concave parts of a shape will not propagate as far out in a distance map as the convex parts. As a consequence points placed on the convex parts of a shape are given more weight than points on concave parts. Our approach differs from the approach presented in [5], as it uses signed distance maps and minimizes the squared difference between the signed distance maps restricted to a shrinking narrow band. Thus, it does not suffer from the same defect as 5 .

\section{Theory}

The registration of a surface $S_{x}$ to a surface $S_{y}$ w.r.t the Euclidean metric can be expressed as the minimization of the functional

$$
F_{1}(\boldsymbol{p})=\oint_{\mathcal{S}_{x}} d\left(W(\boldsymbol{x} ; \boldsymbol{p}), \mathcal{S}_{y}\right)^{2} d \boldsymbol{x},
$$

where $W\left(\_; \boldsymbol{p}\right)$ is the warp function.

A minor flaw with this approach is that the registration of $S_{x}$ to $S_{y}$ is not necessarily equivalent to the registration $S_{y}$ to $S_{x}$. If $W\left(_{-} ; \boldsymbol{p}\right)$ is invertible (1) can be extended to

$$
\begin{aligned}
F_{2}(\boldsymbol{p}) & =\oint_{\mathcal{S}_{x}} d\left(W(\boldsymbol{x} ; \boldsymbol{p}), \mathcal{S}_{y}\right)^{2} d \boldsymbol{x}+\oint_{\mathcal{S}_{y}} d\left(W(\boldsymbol{y} ; \boldsymbol{p})^{-1}, \mathcal{S}_{x}\right)^{2} d \boldsymbol{y} \\
& =\oint_{\mathcal{S}_{x}} d_{, \mathcal{S}_{y}}(W(\boldsymbol{x} ; \boldsymbol{p}))^{2} d \boldsymbol{x}+\oint_{\mathcal{S}_{y}} d_{\mathcal{S}_{x}}\left(W(\boldsymbol{y} ; \boldsymbol{p})^{-1}\right)^{2} d \boldsymbol{y},
\end{aligned}
$$

where $d_{\mathcal{S}_{x}}$ and $d_{\mathcal{S}_{y}}$ are the distance maps of the surfaces $\mathcal{S}_{x}$ and $\mathcal{S}_{y}$, respectively. This energy functional ensures a symmetric registration.

A minimum of $F_{2}$ can be obtained by any gradient or Newton based optimization scheme. However, such schemes may very well get stuck in a local minimum instead of the global minimum. To overcome this problem we introduce a slightly different energy functional

$$
F_{3}(\boldsymbol{p})=\int_{U_{x}^{r}}\left(\Phi_{y}(W(\boldsymbol{x} ; \boldsymbol{p}))-\Phi_{x}(x)\right)^{2} d \boldsymbol{x}+\int_{U_{y}^{r}}\left(\Phi_{x}\left(W(\boldsymbol{y} ; \boldsymbol{p})^{-1}\right)-\Phi_{y}(y)\right)^{2} d \boldsymbol{y}
$$

where $\Phi_{x}(\boldsymbol{x})$ and $\Phi_{y}(\boldsymbol{y})$ are the signed distance maps (SDMs) of the surfaces $\mathcal{S}_{x}$ and $\mathcal{S}_{y}$, and $U^{r}=\left\{\boldsymbol{x}\left|\boldsymbol{x} \in \mathbb{R}^{d},\right| \Phi(x) \mid<r\right\}$. And we note that

$$
F_{3} \rightarrow F_{2} \text { for } r \rightarrow 0 \text {. }
$$




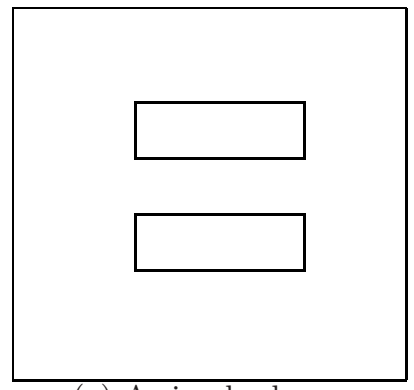

(a) A simple shape.

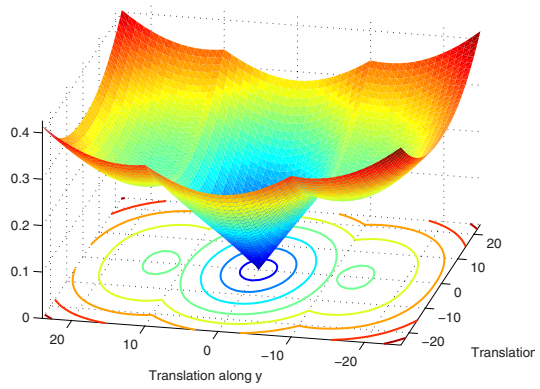

(b) $F_{2}$ cost functional.

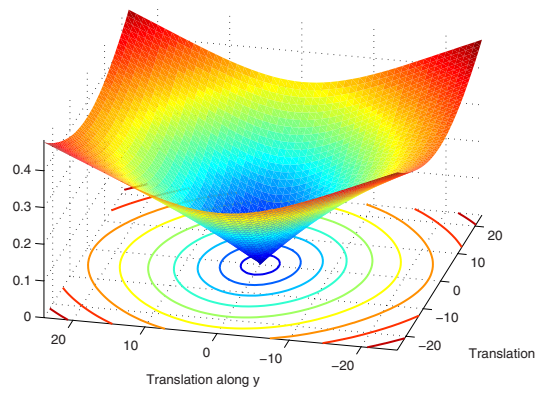

(c) $F_{3}$ cost functional.

Fig. 1. Cost as a function of translation in $x$ and $y$ direction

Now, consider the shape in Figure 1(a) consisting of two identical rectangles. If we translate the shape in both the $x$ and the $y$ direction between -25 and 25 pixels and calculate the energy in each position using $F_{2}$ and $F_{3}$ with $r=25$ pixels, we get energy landscapes shown in Figure $1(b, c)$. In this case, the $F_{2}$ cost function produces three minima while the $F_{3}$ cost function produces only the global minimum.

\section{Method}

The energy functional defined in $F_{3}$ can be viewed as a image registration problem between two $\operatorname{SDMs} \Phi_{x}$ and $\Phi_{y}$, where the points to be warped are those inside the narrow bands $U_{x}^{r}$ and $U_{y}^{r}$. The problem is solved using an extended version of the inverse compositional algorithm presented by Baker et al [1. To preserve the same notation as in [1, we assume that $\Phi_{x}$ and $\Phi_{y}$ are discretized SDMs. Thus, $F_{3}$ becomes

$$
F_{4}(\boldsymbol{p})=\sum_{\boldsymbol{x} \in U_{x}^{r}}\left(\Phi_{y}(W(\boldsymbol{x} ; \boldsymbol{p}))-\Phi_{x}(\boldsymbol{x})\right)^{2}+\sum_{\boldsymbol{y} \in U_{y}^{r}}\left(\Phi_{x}\left(W(\boldsymbol{y} ; \boldsymbol{p})^{-1}\right)-\Phi_{y}(\boldsymbol{y})\right)^{2} .
$$


If the set of warps forms a group the minimization of $F_{4}$ is equivalent to the minimization of

$$
\begin{aligned}
F_{5}(\boldsymbol{p}) & =\sum_{\boldsymbol{x} \in U_{x}^{r}}\left(\Phi_{y}(W(\boldsymbol{x} ; \boldsymbol{p}))-\Phi_{x}(W(\boldsymbol{x} ; \Delta \boldsymbol{p}))\right)^{2} \\
& +\sum_{\boldsymbol{y} \in U_{y}^{r}}\left(\Phi_{x}\left(W(\boldsymbol{y} ; \boldsymbol{p})^{-1}\right)-\Phi_{y}\left(W(\boldsymbol{y} ; \Delta \boldsymbol{p})^{-1}\right)\right)^{2} .
\end{aligned}
$$

with the update rule $W(\boldsymbol{x} ; \boldsymbol{p}) \leftarrow W(\boldsymbol{x} ; \boldsymbol{p}) \circ W(\boldsymbol{x} ; \Delta \boldsymbol{p})^{-1}$. By applying the first order Taylor expansion to (6) we get

$$
\begin{aligned}
F_{5}(\boldsymbol{p}) & \approx \sum_{\boldsymbol{x} \in U_{x}^{r}}\left(\Phi_{y}(W(\boldsymbol{x} ; \boldsymbol{p}))-\Phi_{x}(W(\boldsymbol{x} ; \mathbf{0}))-\nabla \Phi_{x} \frac{\partial W(\boldsymbol{x} ; \mathbf{0})}{\partial \boldsymbol{p}} \Delta \boldsymbol{p}\right)^{2} \\
& +\sum_{\boldsymbol{y} \in U_{y}^{r}}\left(\Phi_{x}\left(W(\boldsymbol{y} ; \boldsymbol{p})^{-1}\right)-\Phi_{y}\left(W(\boldsymbol{y} ; \mathbf{0})^{-1}\right)-\nabla \Phi_{y} \frac{\partial W(\boldsymbol{y} ; \mathbf{0})^{-1}}{\partial \boldsymbol{p}} \Delta \boldsymbol{p}\right)^{2}
\end{aligned}
$$

By taking the derivatives of $F_{5}$ w.r.t. $\Delta \boldsymbol{p}$ and setting them equal to zero we get the update equation

$$
\Delta \boldsymbol{p}=-\boldsymbol{H}^{-1}\left(\sum_{\boldsymbol{x} \in U_{x}^{r}} \boldsymbol{S}_{x}^{\top} \boldsymbol{E}_{\boldsymbol{x}}+\sum_{\boldsymbol{y} \in U_{y}^{r}} \boldsymbol{S}_{y}^{\top} E_{y}\right),
$$

where $\boldsymbol{S}_{x}=\nabla \Phi_{x} \frac{\partial W(\boldsymbol{x} ; \mathbf{0})}{\partial \boldsymbol{p}}, \boldsymbol{S}_{y}=\nabla \Phi_{y} \frac{\partial W(\boldsymbol{y} ; \mathbf{0})^{-1}}{\partial \boldsymbol{p}}, E_{x}=\Phi_{y}(W(\boldsymbol{x} ; \boldsymbol{p}))-\Phi_{x}(\boldsymbol{x})$, $E_{y}=\Phi_{x}\left(W(\boldsymbol{y} ; \boldsymbol{p})^{-1}\right)-\Phi_{y}(\boldsymbol{y})$ and $\boldsymbol{H}=\sum_{\boldsymbol{x} \in U_{x}^{r}} \boldsymbol{S}_{x}^{\top} \boldsymbol{S}_{x}+\sum_{\boldsymbol{y} \in U_{y}^{r}} \boldsymbol{S}_{y}^{\top} \boldsymbol{S}_{y}$. Note that $\boldsymbol{H}^{-1}, \boldsymbol{S}_{x}$ and $\boldsymbol{S}_{y}$ only have to be computed once. A S2SR can be obtained with Algorithm 1.

The best sequence of $r_{i}$ 's is properly highly depended on the problem. We have applied the following scheme with success:

$$
r_{i+1} \approx \frac{r_{i}}{2}
$$

Selecting a suitable initial narrow band $r_{0}$ is however not entirely straight forward. If the choice of $r_{0}$ is too small the algorithm may get stuck in a local

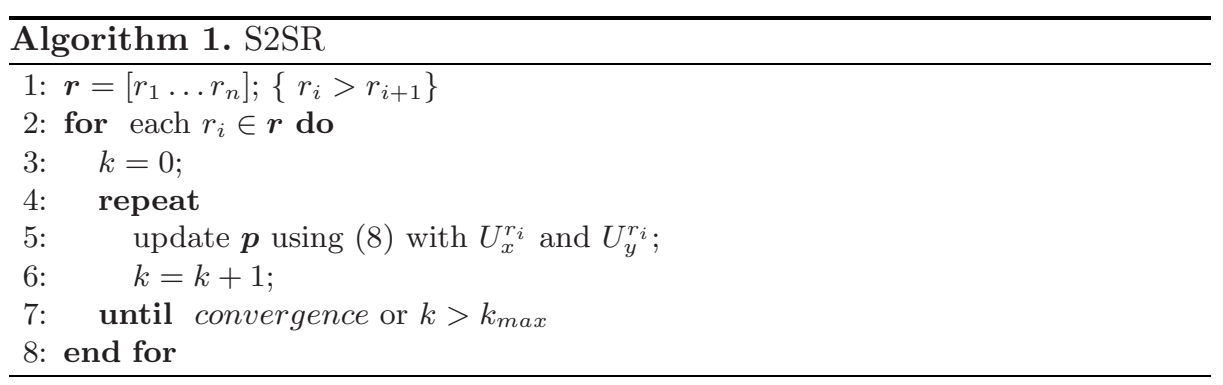


minima, and if it is too large the algorithm will use an unnecessary amount of computational power. Note, that the computation time is much more depended on the radius of the initial narrow band than the number of narrow bands as the global minimum of $F_{3}$ for the narrow band $r_{i}$ properly is relatively close to the global minimum of $F_{3}$ for $r_{i+1}$. In general, $r_{0}$ should be larger than the width of largest structure or feature in the image which might introduce a local minimum in $F_{3}$.

\subsection{Extending Approach to Open Surfaces}

SDMs are in principal only defined for closed surfaces as it is impossible to label the inside and outside of an open surface. Thus, our approach can only be applied to closed surfaces. To overcome this problem we introduce the notion of a pseudo SDM.

The pseudo SDM of triangle mesh of an open surface is computed using the following recipe:

1. Close the surface by triangulating all the holes in the triangle mesh.

2. Compute the SDM of the closed tiangle mesh. Bærentzen et al. 34] describe how to compute the SDM of a closed triangle mesh.

3. Set all voxels in the discretized SDMs with distances to the added faces to an undefined value.

Under the registration, voxels from one SDM may be warped to an undefined volume of the other SDM. In such cases, it is reasonable to assume that the distance in the undefined volume is 0 , as we have no way of knowing whether the point is on the outside or inside of the shape. This hack allows for a bit of slack around the open areas of a surface. In many cases a surface is only open as it has been chosen to disregard a part of the shape - cutting away part of a shape in the exact same place is impossible. Furthermore, the gradient of a SDM at the borders between the defined and undefined volumes is likewise assumed to be equal to 0 .

Sometimes, it is impossible to close an open surface with triangulation without introducing intersections between the new faces and the existing faces. Also, it might not be reasonable to close a surface if the hole is very large. In such cases, it might be more advisable simply to use unsigned distance maps instead of SDMs. The question of how large a hole in a surface can be, before the registration algorithm fails or produces suboptimal result with pseudo SDMs, needs to be investigated in the future.

\section{Experiments}

Two experiments were conducted to test the surface registration approach; (i) a toy example where the outline of the right and left hand of one of the authors were registered to each other, and (ii) a real example where 40 pelvic bones of Duroc pigs were registered with the ICP algorithm by Fitzgibbon [8] and with our S2SR algorithm. 


\subsection{Hand Example}

To test the robustness of the S2SR algorithm a left and a right hand were traced on a piece of paper and scanned into a computer. The left hand was flipped horizontally, displaced 100 pixels in the $x$-direction and -25 pixels in the $y$-direction, and rotated 5 degrees counter clockwise. Figure 2 shows the initial position of the hands, the final position with regular S2SR 1 and the final position with our S2SR algorithm with the narrow bands $r=30,15,7,3,1$. Evidently, the regular S2SR approach gets stuck in a local minimum or saddle point, while the shrinking narrow band S2SR approach registers the left and right hand perfectly.

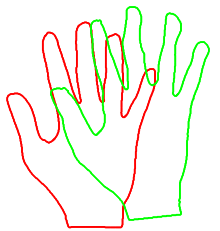

(a) Initial position.

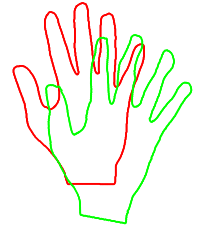

(b) Final position for S2SR with narrow band $\mathrm{r}=1$.

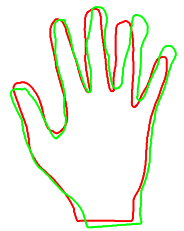

(c) Final position for S2SR with narrow bands 30,15 , $7.5,1$.

Fig. 2. Rigid registration of left (green) and right hand (red)

\subsection{Pelvic Bones}

Half pig skeletons were automatic extracted from CT scans of half pig carcasses and fitted with implicit surfaces. From the implicit surfaces triangle meshes were created, and the pelvic bones were manually removed from the triangulated skeletons. An example of a pelvic bone can be found in Figure 3 .

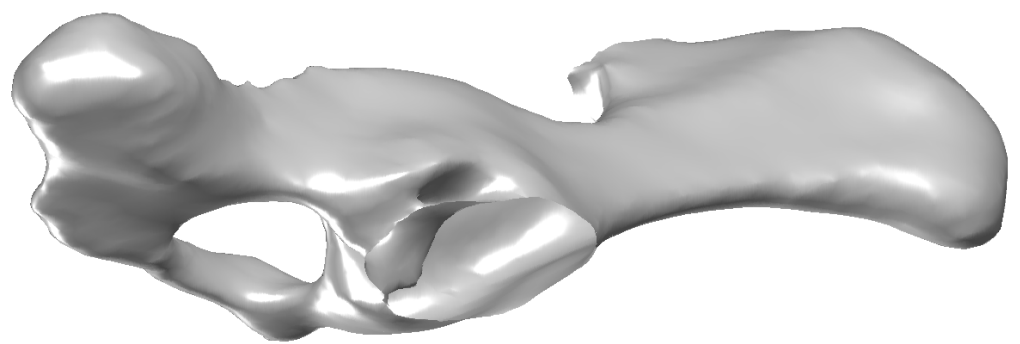

Fig. 3. Example of a pelvic bone from a Duroc pig

From the set of pelvic bone shapes a shape was selected to be the reference, and the remaining shapes were registered to the reference shape with ICP and our level set based S2SR algorithm with $r=20,10,5,2.5,1 \mathrm{~mm}$. To compare

\footnotetext{
${ }^{1}$ Simulated with the small narrow band $\mathrm{r}=1$.
} 
the two registration approaches we use the mean squared error (MSE) and the maximum error (ME). As ICP minimizes the point-to-closest-point (CP) distance and our algorithm minimizes the surface-to-surface distance 2 , we evaluate the performance of the registration algorithms using both distance concepts. Furthermore, as our registration algorithm does symmetric minimization of the squared distances and ICP does not, the MSE and the ME are calculated in the same direction as the ICP registration, in the other direction and in both directions combined. The registration results for ICP and S2SR can be found in Table 1 and 2 respectively. As no surprise, the ICP registration has a lower MSE and ME in the same direction as the registration, when we are using the CP distance. It is neither a surprise that our S2SR algorithm has lower MSEs and MEs in the opposite direction of the ICP registration and in both directions. It is however a bit of a surprise, that our S2SR algorithm has a smaller MSE than ICP when using the SDMs to extract distances. A possible explanation for this result is that our algorithm allows for a bit of slack around the open regions of the surface and is therefore better at fitting the remaining regions of the surface. Figure 4 illustrates this by color-coding the surfaces of two registered pelvic bones with the shortest distance.

Table 1. The MSE and ME averaged over the 39 registrations after ICP registration

\begin{tabular}{|c|c|c|c|c|c|c|}
\hline Method & \multicolumn{5}{|c|}{$\mathrm{ICP}(A \rightarrow B)$} \\
\hline Direction & \multicolumn{2}{|c|}{$A \rightarrow B$} & \multicolumn{2}{|c|}{$A \leftarrow B$} & \multicolumn{2}{|c|}{$A \leftrightarrow B$} \\
\hline Measure & $\sqrt{M S E}$ & $M E$ & $\sqrt{M S E}$ & $M E$ & $\sqrt{M S E}$ & $M E$ \\
\hline SDM & 11.92 & 20.13 & 12.70 & 27.04 & 12.34 & 27.24 \\
\hline CP & 12.32 & 20.85 & 14.40 & 29.41 & 13.44 & 29.48 \\
\hline
\end{tabular}

Table 2. The MSE and ME averaged over the 39 registrations after S2S registration

\begin{tabular}{|c|c|c|c|c|c|c|}
\hline Method & \multicolumn{5}{|c|}{ S2SR $(A \leftrightarrow B)$} \\
\hline Direction & \multicolumn{2}{|c|}{$A \rightarrow B$} & \multicolumn{2}{|c|}{$A \leftarrow B$} & \multicolumn{2}{c|}{$A \leftrightarrow B$} \\
\hline Measure & $\sqrt{M S E}$ & $M E$ & $\sqrt{M S E}$ & $M E$ & $\sqrt{M S E}$ & $M E$ \\
\hline SDM & 11.69 & 24.11 & 12.18 & 24.03 & 11.90 & 26.28 \\
\hline CP & 12.77 & 25.70 & 13.11 & 26.34 & 12.95 & 28.30 \\
\hline
\end{tabular}

W.r.t. computation time, it can be mentioned that it took approximately 20 minutes to run the 39 registrations with ICP and approximately 50 minutes to run 39 registrations with $\mathrm{S} 2 \mathrm{SR}$ on a standard Dell laptop with a 1.6Ghz Centrino $\mathrm{CPU}$ and $2 \mathrm{~Gb}$ ram. It is difficult to compare the computation time of the two algorithm as the computation time for the S2SR algorithm is vastly depended

\footnotetext{
${ }^{2}$ The distances are found by interpolating the SDMs. To ensure fairness, when evaluating the MSE and ME, points, which are warped to an undefined area of a SDM, are ignored instead of receiving the distance 0 .
} 

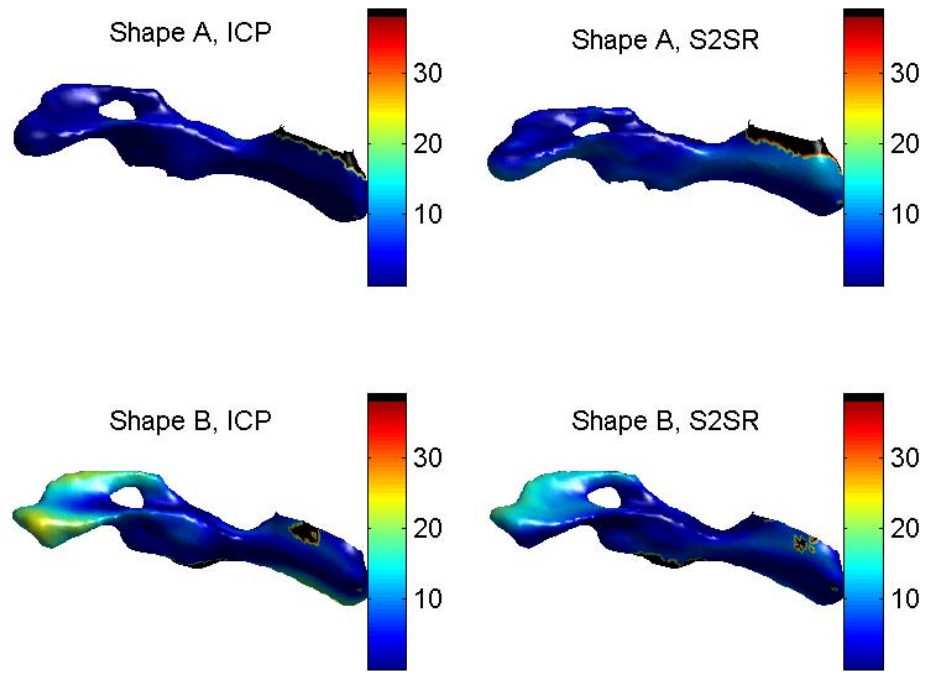

Fig. 4. Distance color-coded surfaces after registration. Black areas are areas where the distance could not be interpolated in the SDM because the point is situated in a undefined area.

on the chosen parameters, e.g. the chosen narrow bands and the resolution of the discretized SDMs.

\section{Conclusion}

This paper has presented a method for S2SR. The registration algorithm was tested on two examples, where its properties were highlighted; (i) it is less prone to fall into local minima than ordinary S2SR, (ii) and it does symmetric registration. As the method relies on SDMs it only works in theory on surfaces. Nevertheless, this paper has demonstrated that it can work on open surfaces by introducing a pseudo SDM, where distances are not defined in volumes close to the open regions of the surface.

In the future, we will use non-rigid transformations with the registration approach.

\section{References}

1. Baker, S., Matthews, I.: Lucas-Kanade 20 Years On: A unifying Framework". CMU. Part 1 (2002)

2. Besl, P.J., Mckay, N.D.: A Method for Registration of 3-D Shapes. IEEE Transaction on Pattern Analysis and Machine Intelligence 14, 239-256 (1992)

3. Bærentzen, A.: Robust Generation of Signed Distance Fields from Triangle Meshes. Fourth International Workshop on Volume Graphics (2005) 
4. Bærentzen, A., Aanæs, H.: Signed Distance Computation using the Angle Weighted Pseudo-normal. IEEE Transactions on Visualization and Computer Graphics, 11, 243-253 (2005)

5. Darkner, S., Vester-Christensen, M., Larsen, R., Nielsen, C., Paulsen, R.R.: Automated 3D Rigid Registration of Open 2D Manifolds, MICCAI 2006 Workshop From Statistical Atlases to Personalized Models (2006)

6. Davies, R.H., Twining, C.J., Cootes, T.F., Waterton, J., Taylor, C.J.: A minimum description length approach to statistical shape modelling, IEEE Transactions on Medical Imaging (2002)

7. Dryden, I.L., Mardia, K.V.: Statistical Shape Analysis. John Wiley, New York (1998)

8. Fitzgibbon, A.: Robust Registration of 2D and 3D Point Sets. In: Proc. British Machine Vision Conference. vol. II (2001)

9. Gower, J.C.: Generalized procrustes analysis. Psychometrika (1975)

10. S. Granger, X. Pennec. "Multi-scale EM-ICP: A Fast and Robust Approach for Surface Registration." ECCV 2002, 2002.

11. Rusinkiewicz, S., Levoy, M.: Efficient Variants of the ICP Algorithm. 3DIM 2001 (2001) 